\title{
Teaching a Humanoid: A User Study with HOAP-3 on Learning by Demonstration
}

\author{
Astrid Weiss, Judith Igelsböck, Sylvain Calinon (*), Aude Billard (*), Manfred Tscheligi (*)
}

(*) Member, IEEE

\begin{abstract}
This article reports on the results of a user study investigating the satisfaction of naïve users conducting two learning by demonstration tasks with the HOAP-3 robot. The main goal of this study was to gain insights on how to ensure a successful as well as satisfactory experience for naïve users. Participants performed two tasks: They taught the robot to (1) push a box, and to (2) close a box. The user study was accompanied by three pre-structured questionnaires, addressing the users' satisfaction with HOAP-3, the user's affect toward the robot following from the interaction, and the user's attitude towards robots. Furthermore, a retrospective think aloud was conducted to gain a better understanding of what influences the users' satisfaction in learning by demonstration tasks. The results stress that learning by demonstration is a promising approach for naïve users to learn the interaction with a robot, as a high task completion and final satisfaction rate could be observed. Moreover, the short term interaction with HOAP-3 led to a positive affect higher than the normative average on half of the female users.
\end{abstract}

\section{INTRODUCTION}

An increasing presence of robots in future society will lead to an increased number of naïve users faced with interaction and collaboration with robots [2]. When robots are introduced into working and living environments naïve users will have to be able and willing to teach the robots different tasks according to changing requirements. Robot's tasklearning is usually focused on usability issues like efficiency [6]. We stress the importance of user satisfaction and positive affect to attain a human's willingness to conduct learning by demonstration tasks with robots. Consider a robot assisting in solving tasks at an assembly line or in a canteen kitchen. The robot should be able to learn various tasks and perform them appropriately, even by demonstration of users without pre-knowledge in robotics technology. Robotic agents therefore require a high degree of intuitiveness on the user's side and should not cause immediate frustration in the initial teaching phase. We propose a learning by demonstration scenario based on interactive tutelage whereby the user embodies the robot during training by moving kinesthetically the robot's arms. This offers the user with natural easy-to use means of

Manuscript received March 25, 2009. This work was supported in part by the ROBOT@CWE project (funded by FP6-2005- IST-5).

A. Weiss, Judith Igelsböck, and M. Tscheligi are with the ICT\&S Center, University of Salzburg, Sigmund-Haffner-Gasse 18, 5020 Salzburg, Austria (phone: +43-662-8044-4812; fax: +43-662-8044-4800; e-mail: ( \{astrid.weiss, judith.igelsboeck, manfred.tscheligi\}@ sbg.ac.at).

S. Calinon and A. Billard are with the LASA Laboratory, EPFL, CH1015 Lausanne, Switzerland (e-mail: aude.billard@epfl.ch; sylvain.calinon@epfl.ch). teaching the robot, which we hypothesized would subsequently result in more satisfaction and, thus, positive affect toward the particular teaching situation. While robot programming by demonstration has always claimed to develop natural means of interacting with the robot [5] [13] very few studies have actually measured how satisfying these means really were for potential users, exceptions are [15] [8] [19]. The present study emphasizes the human's perspective and needs when teaching a humanoid robot, namely Fujitsu HOAP-3 robot. The human's task performances, emotions and attitude towards the HOAP-3 robot will be investigated. The main research goal was to determine whether this form of embodied tutelage would allow naïve users to teach successfully the task, while making it a satisfactory experience. We thus combine both a quantitative analysis based on various measures of task performance and a qualitative would analysis of the humanrobot interaction by means of a retrospective think aloud.

The work is presented as follows: First, theories and results from former user studies are discussed. Second, the research questions, the study setting, and the methodical approach are described, followed by the results of the user study. The last part of the presented work is dedicated to the lesson learned and future work.

\section{RELATED WORK}

\section{A. Learning by demonstration}

Learning by demonstration is one particular machine learning technique, where a policy is learned from examples provided by a teacher [2] [4]. The robot recodes and reproduces the demonstrated actions. This is in contrast to other techniques, e.g. reinforcement learning where a policy is obtained by exploration. As learning from demonstration is an intuitive communication medium for humans and does not require expert knowledge, it is appropriate as well for experts as non-experts of robotics.

\section{B. Human teaching behavior}

Thomas and Breazeal [18] stress that people develop a mental model of the learner; they use the reward channel for both guidance and feedback. Furthermore, they require a separate channel for motivational feedback.

Investigating the characteristics a robot needs for efficient work with a human partner Breazeal, Hoffman and Lockerd [6] base on joint intention and learning theory [22] [11] [12]. They assume that humans are used to teach in a social and collaborative way and usually choose the dialog as a form for tutelage. [12] emphasizes the importance of 
confirmations for mutual agreement on accomplishment of a task and [6] highlight the importance of making the collaborative work with robots not only efficient but also enjoyable for humans, by using social skills.

\section{Studying Human-Robot-Learning Scenarios}

Several studies have been conducted to increase the naturalness and intuitiveness of the human teacher - robot learner interaction in a social manner. In doing so, most of prior work concentrated on technical issues [e.g. [3] [10] [14] [16] [17] [21]. Only a few studies focused on the users' perceptions and expectations regarding the competences of the robot. For instance, [20] studied the human role when teaching assistive robots new tasks. They found out that people fall in a positive bias in rewards deriving from the opinion that the learner needs to be motivated and encouraged for better learning. [1] analyzed imitation as learning tool in human robot interaction in a pilot user study comparing the machine centric and the human centric perspective. They identified a good alignment between the human centered assessment and the system centered measurement of the imitative performance. In both studies the users were not offered a human-robot interaction situation with a physically present humanoid robot as within the presented work.

\section{THE UsER STUdy}

\section{A. Study Setting}

The study took place at the Learning Algorithms and Systems Laboratory (LASA), EPFL Lausanne, Switzerland from 10th to 15th of August, 2008.

12 participants (eight females, four males) took part in the user study. The average age of the participants was 26.58 years; the youngest participant was aged 16, the oldest participant was aged 40 . The requirements upon which the participants were selected were: (1) good knowledge of English (the study was conducted in English) and (2) no technical experience related to robotics (since we aimed at assessing the ability of naive users to carry out the tasks).

\section{B. Study Procedure}

The study procedure was split into three parts:

1) Pre-interview and briefing

The first step was conducted in a separate room before the participants could see the robot. They were welcomed and introduced into the scenario and the procedure of the user study.

\section{2) Task completion}

Subsequently the participants were introduced to HOAP-3. They were shown how the robot's arm can be moved and where to put the box. Then they conducted the two tasks together with the robot. Directly after each tasks the participants were asked to talk about their thoughts, feelings, and problems during their interaction with the robot and gave suggestions on how the learning by demonstration procedure could be made easier for the future (retrospective think aloud).

\section{3) Pre-structured questionnaires}

After the task completion the participants had to fill in two pre-structured questionnaires on satisfaction and affect.

\section{Experimental procedure}

The experiment consisted in two tasks, during which participants had to teach the humanoid HOAP-3 robot from Fujitsu. As mentioned before, teaching relied on an interactive tutelage scenario whereby the user would embody the robot by placing his or her arms around the robot and guide it through the motion. After each training trial, the robot would reproduce the task at the best of its knowledge. Based on visual assessment of the successfulness of the reproduction the user could then choose if ( $\mathrm{s}$ )he wanted to retrain the robot once more.

In the first task, the participants had to teach the robot to push a box, moving it toward the user (Fig.1.); in the second task, the job was to teach the robot to close the same box by pushing on the box's cover (Fig.2.). These were simple tasks, chosen to require no particular skill on the trainer and so that they would be performed likely the same way by all trainers.

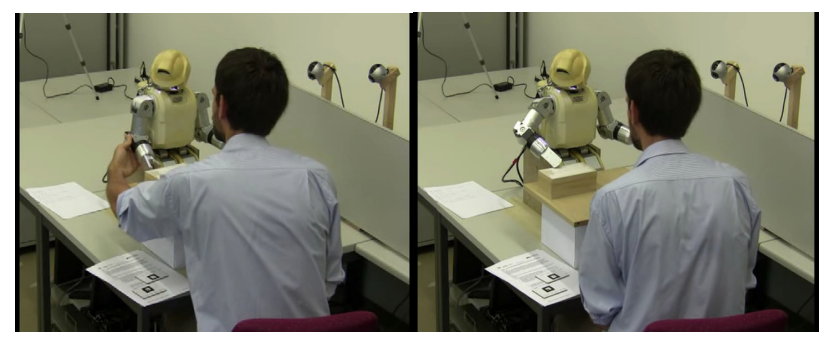

Fig.1. Demonstration and Repetition of task 1 "Push the box"

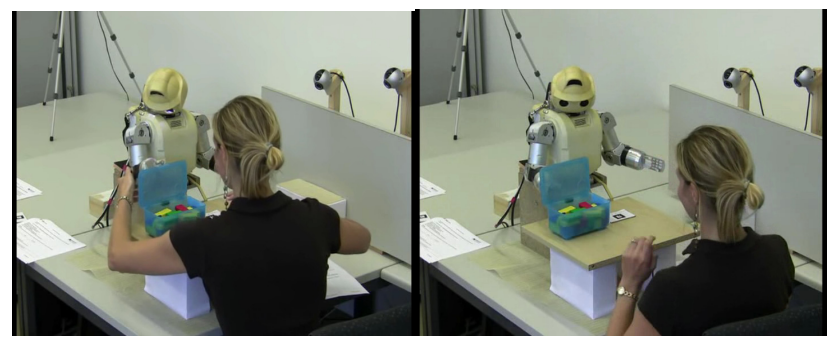

Fig.2. Demonstration and Repetition of task 2 "Close the box"

To prevent a carryover effect from one task to the other as well as a difference in satisfaction rating because of the task difficulty, the order in which tasks were accomplished was counter-balanced. In condition 0 , the task "Close the box" had to be accomplished first, and in condition 1, the task "Push the box" was the first to be accomplished. Participants were introduced to the scenario with the following text:

"Imagine you are working at an assembly line in a big fabrication plant. A new robot is introduced, which should support you in solving tasks. You can teach the robot specific motions by demonstrating them (meaning move the robot's arm like you expect it to move it later on its own); the robot will repeat the learnt motion. You can repeat this 'demonstration-repetition-cycle" for as long until you are pleased with the result." 
Afterwards the participants received the instructions on how to teach HOAP-3. Table I gives the exact wording of how these two tasks were presented to the participants.

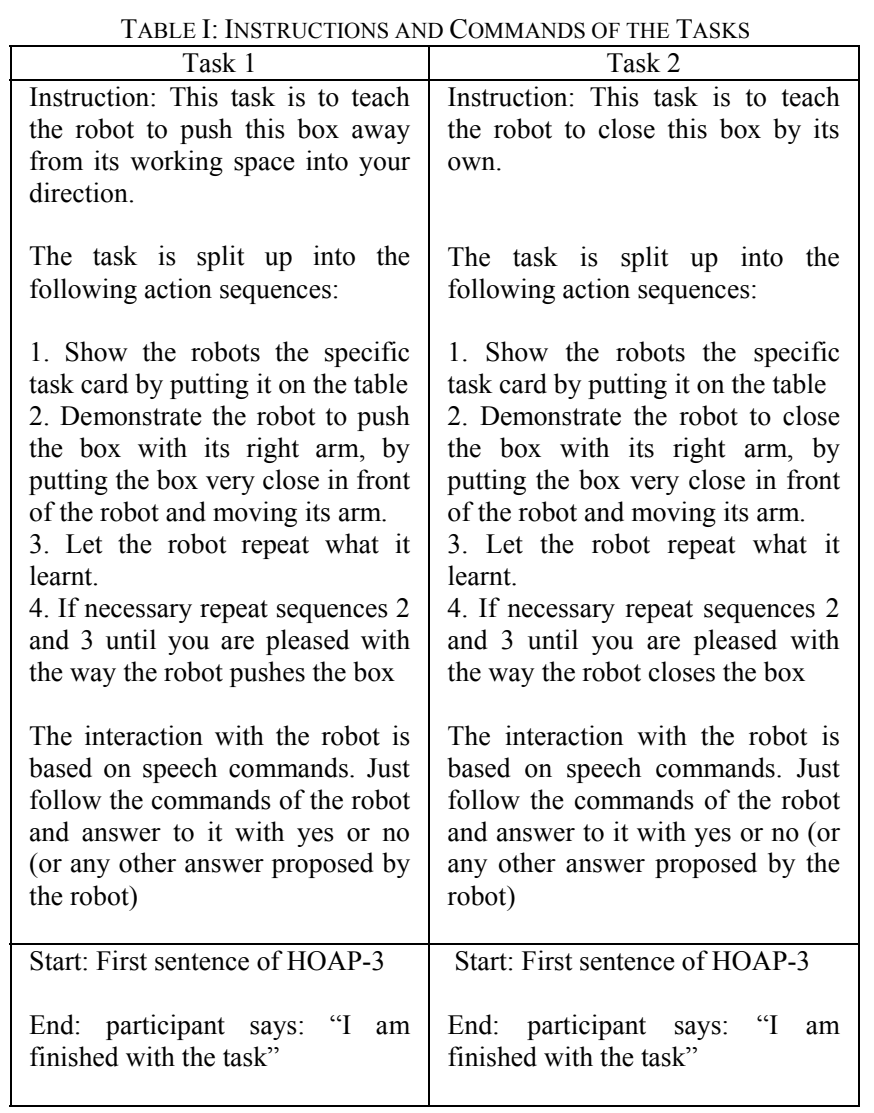

The main focus of the study setting was to let the participants decide for themselves (1) how many times they demonstrated the arm movement of HOAP-3 until they were satisfied and (2) how many times they let the robot repeat the learnt task until they were satisfied with the result.

Thus, a task was counted as carried out successfully if (1) the robot closed/ pushed the box and (2) if the user stated I am finished (maximum time given to the participants was 10 minutes).

After giving the participants the instructions on how to teach the robot, they were sat in front of the robot and interacted with it by executing its speech commands and manipulating its arm via direct contact interaction (see Fig. 1. and 2.). A wizard-of-Oz method was chosen to avoid speech recognition errors. The operator stood behind the participant and operated the robot using a wireless Wii remote control and thus was invisible to the participants. The operator performed the following commands when needed:

1) Provide yes/no answers to the robot

2) Move to the next phase of the interaction

3) Abort the current movement and re-start the current phase

Figure 3. shows the dialogue structure for the study setting.

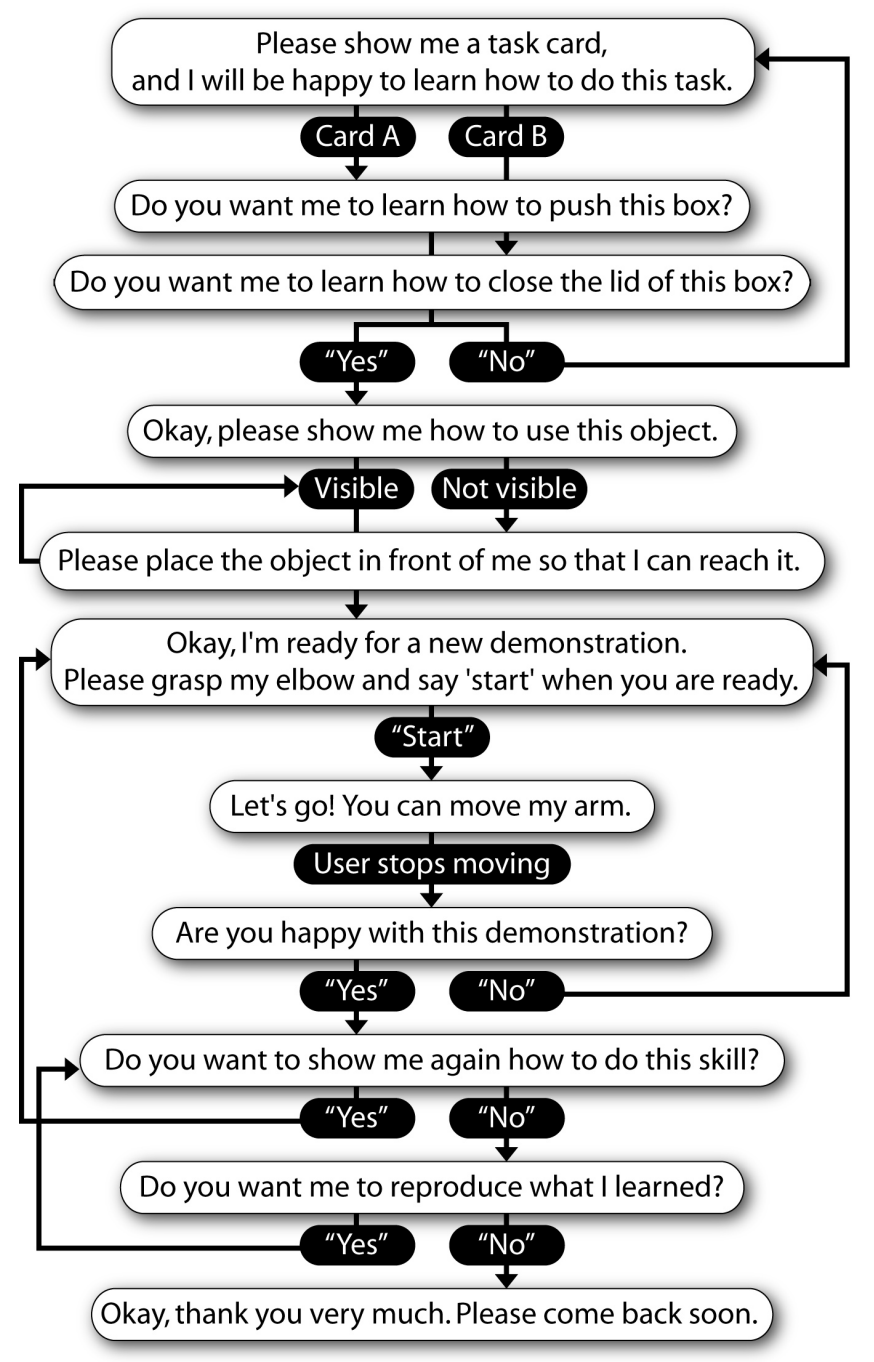

Fig. 3. Dialogue Structure

\section{Technical Set-up}

During training, the robot records the position of its arm and torso joints and of its hands. Simultaneously, two external cameras placed on the side of the robot are used to locate the object in $3 \mathrm{D}$ by tracking visual patches attached to it. The set of joint and hands' trajectories is incrementally encoded in a Gaussian Mixture Model (GMM). After each demonstration, a generalized version of the trajectories is reproduced through Gaussian Mixture Regression (GMR). Through the use of the compact GMM representation, the robot can extract the essential characteristics of the demonstrations autonomously. The regression process is then used to retrieve a controller satisfying several constraints simultaneously (represented either in joint space or in task space), see [9] for details. Note that the user can place the object at a different location during the reproduction attempts, and that the robot for each iteration re-computers an optimal controller to satisfy as best as possible the constraints extracted during the demonstrations.

\section{E. Goals and Research Questions}

The following research questions were investigated by means of the presented user study: 
RQ1: Can naïve users successfully teach such simple tasks to the robot without need of particular training?

RQ2: Would the user's satisfaction worsen as an effect of having to repeat the training several times?

RQ3: Does the perceived difficulty of the tasks influence the general system satisfaction?

RQ4: Does the interaction with HOAP-3 stimulate a positive or negative affect for the user?

\section{F. Methodological Approach}

The user study was based on the USUS evaluation framework, which was developed to support a holistic evaluation of human-robot interaction in terms of usability, social acceptance, user experience, and societal impact [24]. Based on this framework the pre-structured questionnaires were chosen for the user study.

In order to capture data on the demonstration count and participants' comments while interacting with the robot the whole user study was taped on video. Furthermore, after each tasks the participants were asked on how difficult they experienced the movement to be taught.

\section{1) Satisfaction Measures}

Besides recording the task duration and completion rate to see if naïve users can successfully carry out the learning by demonstration tasks, a count on the users' demonstrations and the robot's repetitions was noted. Furthermore the System Usability Scale (SUS) [7] questionnaire was used. This is a validated, standardized questionnaire used mainly in HCI to quickly asses the overall satisfaction with a computing system. The result of this questionnaire is one overall value representing general satisfaction with the system. The SUS score can range from "0-100": "80-100: Users like the system", "60-79: Users accept the system" and "0-59: Users do not like the system".

2) Affect Measure

To address the affect the interaction with HOAP-3 had on the users, the PANAS questionnaire [23] was used. PANAS

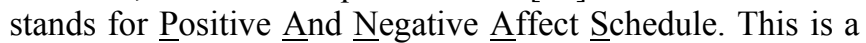
20 items self-report measure of positive and negative affect developed by [23]. The PANAS questionnaire has been used to measure participants' current emotional state, directly after interacting with the robot. A highly negative affect value (NA) indicates distress and non pleasurable engagement. By contrast a highly positive affect value (PA) represents a pleasurable experience engagement with the robot.

\section{Results}

\section{A. Task Performance and User Satisfaction}

Tables II and III give an overview on task completion, task duration, number of demos a participant showed to the robot, number of repetitions the robot showed, with how many demos the participants was satisfied and the final satisfaction with the way HOAP-3 repeated the learnt motion.
TABLE II: "CLOSE THE BOX"

\begin{tabular}{|l|l|l|l|l|l|l|}
\hline TP & $\begin{array}{l}\text { total } \\
\text { dur. }\end{array}$ & $\begin{array}{l}\text { task } \\
\text { compl. }\end{array}$ & $\begin{array}{l}\text { demo } \\
\text { count }\end{array}$ & $\begin{array}{l}\text { satisfied } \\
\text { demos }\end{array}$ & $\begin{array}{l}\text { repetition } \\
\text { count }\end{array}$ & $\begin{array}{l}\text { final } \\
\text { satis. }\end{array}$ \\
\hline 1 & $2: 52$ & yes & 3 & 0 & 2 & no \\
\hline 2 & $2: 49$ & yes & 2 & 1 & 2 & yes \\
\hline 3 & $10: 14$ & no & 4 & 1 & 4 & no \\
\hline 4 & $1: 59$ & yes & 1 & 1 & 2 & yes \\
\hline 5 & $5: 02$ & yes & 5 & 2 & 2 & yes \\
\hline 6 & $3: 04$ & yes & 3 & 1 & 1 & yes \\
\hline 7 & $5: 28$ & yes & 4 & 3 & 3 & yes \\
\hline 8 & $3: 46$ & yes & 4 & 1 & 1 & yes \\
\hline 9 & $5: 30$ & yes & 5 & 4 & 4 & yes \\
\hline 10 & $2: 44$ & yes & 1 & 1 & 2 & yes \\
\hline 11 & $2: 24$ & yes & 1 & 1 & 2 & yes \\
\hline 12 & $10: 45$ & yes & 11 & 7 & 12 & yes \\
\hline
\end{tabular}

TABLE III: "PUSH THE BOX"

\begin{tabular}{|l|l|l|l|l|l|l|}
\hline TP & $\begin{array}{l}\text { total } \\
\text { dur. }\end{array}$ & $\begin{array}{l}\text { task } \\
\text { compl. }\end{array}$ & $\begin{array}{l}\text { demo } \\
\text { count }\end{array}$ & $\begin{array}{l}\text { satisfied } \\
\text { demos }\end{array}$ & $\begin{array}{l}\text { repetition } \\
\text { count }\end{array}$ & $\begin{array}{l}\text { final } \\
\text { satis. }\end{array}$ \\
\hline 1 & $4: 05$ & yes & 3 & 3 & 3 & no \\
\hline 2 & $3: 56$ & yes & 2 & 2 & 2 & yes \\
\hline 3 & $1: 55$ & yes & 1 & 1 & 2 & yes \\
\hline 4 & $2: 57$ & yes & 2 & 1 & 2 & yes \\
\hline 5 & $2: 49$ & yes & 2 & 1 & 3 & yes \\
\hline 6 & $1: 38$ & yes & 1 & 1 & 1 & yes \\
\hline 7 & $4: 02$ & yes & 3 & 2 & 2 & yes \\
\hline 8 & $3: 27$ & yes & 2 & 2 & 3 & yes \\
\hline 9 & $2: 19$ & yes & 2 & 1 & 1 & yes \\
\hline 10 & $6: 35$ & yes & 5 & 5 & 5 & yes \\
\hline 11 & $4: 01$ & yes & 4 & 4 & 4 & yes \\
\hline 12 & $6: 01$ & no & 8 & 4 & 4 & no \\
\hline
\end{tabular}

The tables show that 11 participants could successfully carry out task to close the box and 11 participants to push the box. TP3 was able to teach the robot to close box, but was not satisfied with it in the end. TP12 could not teach the robot to push the box and even though time was left to teach it, the participant aborts the task because of frustration. However, TP3 improved his/her teaching skills and solved the second task successfully and satisfyingly in only 1:55 and TP12 managed to solve his/her second task as well successfully and satisfyingly (but TP12 exceeded the time frame of 10 minutes).

No statistical change could be found regarding task duration or demonstration count from the first to the second task. In other words participants did not get faster in teaching nor made use of less demonstration in the second task.

11 out of 12 participants were finally satisfied with the repetition of the robot to close the box. 10 out of 12 participants were satisfied with how the robot finally pushed the box. However, no statistical significant correlation could be found between the number of demonstration the participants showed and their final satisfaction (task 1: -0.33 , task 2: -0.506) as well as the number of repetitions and their final satisfaction (task 1: -0.208 , task 2: -367). 
TABLE IV: SUS AND PERCEIVED DIFFICULTY

\begin{tabular}{|l|l|l|l|}
\hline TP & SUS & $\begin{array}{l}\text { Rating } \\
\text { Close box }\end{array}$ & $\begin{array}{l}\text { Rating } \\
\text { Push Box }\end{array}$ \\
\hline 1 & 65 & 2 & 5 \\
\hline 2 & 57,5 & 2 & 1 \\
\hline 3 & 55 & 5 & 1 \\
\hline 4 & 70 & 3 & 3 \\
\hline 5 & 57,5 & 4 & 3 \\
\hline 6 & 85 & 2 & 1 \\
\hline 7 & 82,5 & 2 & 4 \\
\hline 8 & 40 & 2 & 4 \\
\hline 9 & 55 & 2 & 4 \\
\hline 10 & 70 & 2 & 4 \\
\hline 11 & 72,5 & 1 & 4 \\
\hline 12 & 80 & 4 & 4 \\
\hline
\end{tabular}

The SUS revealed scores between 40 and 85 (table IV). Thus, three participants liked the system, four participants accepted the system, and five did not like the system. However no significant correlation could be found between the SUS score and the rating of difficulty or the numbers of demos/ repetitions.

The feeling of (dis)satisfaction was also reflected in the think aloud data by the participants. 35 negative statements and 31 positive statements were extracted as well as 17 suggestions for improvements.

The participants were mainly unsatisfied due to a lack of technical background information ("I did not understand why he did not see the card", "I did not understand where its range of vision is", "He easily found the task card but I do not know why"). The participants did not like if things happened, they could not explain to themselves which could have been avoided if the robots capabilities were introduced in educational lessons beforehand. Other negative comments were on fears of contact with the robot ("I was afraid the robot could get stuck", "I was afraid to do something wrong") and communication problems ("I did not understand what the robot wants", "I is not clear why it says "start"). Improvements suggestions were mainly on fixing the starting position of the box and the arm of the robot ("Mark the place where the box should stand", "A starting position for the robot would be helpful").

\section{B. Positive and Negative Affect}

The PANAS questionnaire revealed that in general female participants showed a positive affect higher than the normative female sample (see).

Three of the six female participants had a considerably more positive affect after interacting with HOAP-3 (TP5 $=36$, $\mathrm{TP} 7=37, \mathrm{TP} 8=35)$ than the normal female average (30.6). The other three female participants showed average values around 30. Furthermore the negative affect for women were for all except one (TP9) lower than the normative female sample (16.68, see Fig. 4.)

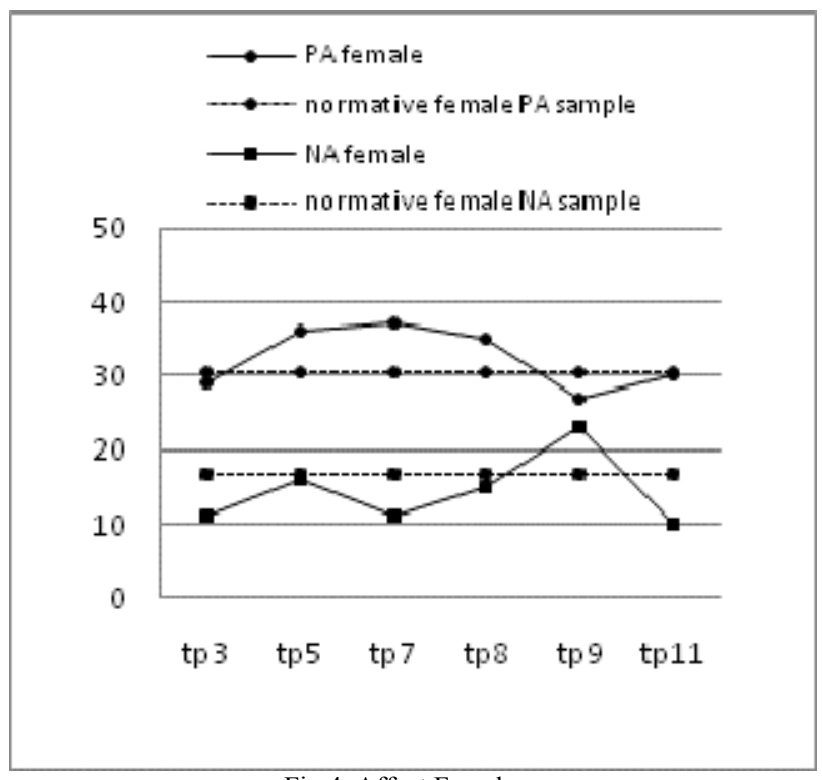

Fig.4. Affect Female

The male participants stated a positive affect slightly under the normative male average (32.06, see Fig. 5.), but also the negative affect was slightly under the normative male average (15.2) as well.

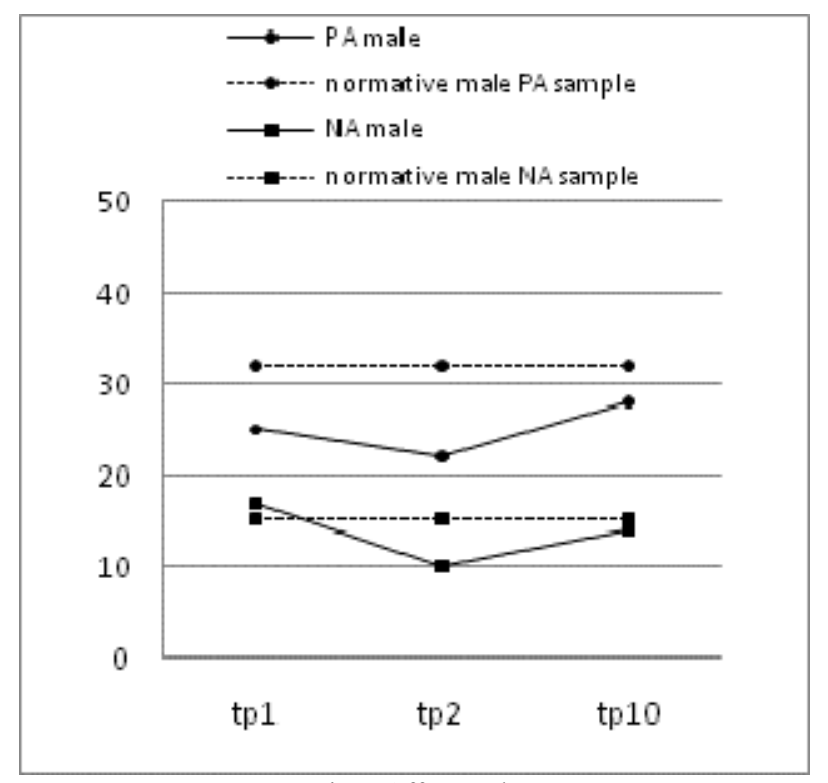

Fig.5. Affect Male

These results indicate that the developed HOAP-3 interactive teaching behavior raises a positive affect above all for women. This fact leads us to the assumption that learning by demonstration with a humanoid robot could be a valuable interaction mode especially for women in long term human-robot interaction scenarios.

\section{Discussion AND CONCLUSION}

The results of the presented work show that learning by demonstration based on direct contact interaction and speech commands is a valuable approach to bring naïve users closer to robotic systems. Except for two participants all were able to successfully carry out the teaching task with the robot and 
except for two participants all were satisfied with the final repetition of the robot (RQ1). Furthermore, we could show that participants are still willing to show the robot several demos to achieve a satisfying repetition result even though they did not become faster in the demonstrations (RQ2).

However as task duration did not no statistically decrease it seems that learning by demonstration is on the one hand a mental model which is easily understood by naïve users, but on the other hand difficult to transfer. Reasons therefore mentioned by the participants were: "I did not know in which way I can move the arm", "I did not know what the robot can see", "I did not know the force of the robot". This lack of background information caused fears of contact and misunderstanding of the robots behavior. Thus it is recommendable providing basic technical background information beyond demonstrating the movement possibilities of the arm also to naïve users (e.g. explaining DoF and the vision system).

A further indicator for the value of learning by demonstration with naïve users was that the perceived difficulty of the tasks did not influence the participants' general satisfaction with the system (no correlation between SUS scale and rating of task difficulty). This indicates that naïve users give the robot a second chance even though they perceived a previous teaching attempt as difficult or unsatisfying. This was also proven by the fact that participants 3 and 12 conducted their second task successfully and were pleased with the result (RQ3). Furthermore, a positive affect towards the HOAP-3 robot after the interaction by means of the PANAS questionnaire could be shown (RQ4).

The results from the retrospective think aloud stressed the importance of ease and intuitiveness of use, but also the "humanness" of the robot when acting as a working colleague was conceived positively in the teaching by demonstration scenario (but clearly this result was biased by the study setting). In general this user study provides first valuable insights into the experiences of naïve users when interacting with a humanoid robot by the means of embodied tutelage.

\section{ACKNOWLEDGMENT}

The user study was conducted in the framework of the Robot@cwe project (funded by FP6-2005- IST-5).

\section{REFERENCES}

[1] Alissandrakis, A.; Nehaniv, C. L.; Dautenhahn, K. \& Saunders, J. Evaluation of robot imitation attempts: comparison of the system's and the human's perspectives HRI '06: Proceedings of the 1st ACM SIGCHI/SIGART conference on Human-robot interaction, ACM, 2006, 134-141.

[2] Argall, B.; Chernova, S.; Veloso, M. \& Browning, B. A survey of robot learning from demonstration Robotics and Autonomous Systems, Elsevier, 2008.

[3] Atkeson, C.G.; Joshua G. Hale, Frank Pollick, Marcia Riley, Shinya Kotosaka, Stefan Schaal, Tomohiro Shibata, Gaurav Tevatia, Ales Ude, Sethu Vijayakumar, Mitsuo Kawato, "Using Humanoid Robots to Study Human Behavior," IEEE Intelligent Systems, vol. 15, no. 4, pp. 46-56, July/Aug. 2000, doi:10.1109/5254.867912
[4] Billard, A.; Calinon, S.; Dillmann, R.; Schaal, S.; Siciliano, B. \& Khatib, O. Robot programming by demonstration Handbook of Robotics, Springer, 2008, 1371-1394.

[5] Breazeal, C. L. Sociable machines: expressive social exchange between humans and robots Massachusetts Institute of Technology, 2000

[6] Breazeal, C.; Hoffman, G. \& Lockerd, A. Teaching and Working with Robots as a Collaboration AAMAS '04: Proceedings of the Third International Joint Conference on Autonomous Agents and Multiagent Systems, IEEE Computer Society, 2004, 1030-1037

[7] Brooke, J. SUS: a" quick and dirty" usability scale Usability evaluation in industry, 1996, 189-194

[8] Calinon S. and Billard A.. What is the teacher's role in robot programming by demonstration? - Toward benchmarks for improved learning. Interaction Studies. Special Issue on Psychological Benchmarks in Human-Robot Interaction, 8(3), 2007

[9] Calinon, S.; Guenter, F. \& Billard, A. On Learning, Representing, and Generalizing a Task in a Humanoid Robot IEEE TRANSACTIONS ON SYSTEMS MAN AND CYBERNETICS PART B, 2007, 37, 286

[10] Chernova, S. \& Veloso, M. Multi-thresholded approach to demonstration selection for interactive robot learning Proceedings of the $3 \mathrm{rd}$ ACM/IEEE international conference on Human robot interaction, 2008, 225-232

[11] Cohen, P. R.; Levesque, H. J.; Nunes, T. and S. L. Oviatt. Taskoriented dialogue as a consequence of joint activity. In Proceedings of the Pacific Rim International Conference on Artificial Intelligence, Nagoya, Japan, November 1990.

[12] Grice, H. P. Logic and conversation. In P. Cole and J. L. Morgan, editors, Syntax and Semantics: Vol. 3: Speech Acts, pages 41-58. Academic Press, San Diego, CA, 1975.

[13] Matarić, M. J. Behavior-Based Robotics as a Tool for Synthesis of Artificial Behavior and Analysis of Natural Behavior, Trends in Cognitive Science, 2(3), Mar 1998, 82-87.

[14] Nehmzow, U.; Akanyeti, O.; Weinrich, C.; Kyriacou, T. \& Billings, S. Robot programming by demonstration through system identification IEEE/RSJ International Conference on Intelligent Robots and Systems, 2007. IROS 2007, 2007, 801-806

[15] Nicolescu M. N. and Matarić, M. J. Natural methods for robot task learning: Instructive demonstrations, generalization and practice. In Proc. of the 2nd Intl. Conf. AAMAS, 2003.

[16] Rybski, P.; Yoon, K.; Stolarz, J. \& Veloso, M. Interactive robot task training through dialog and demonstration

Proceedings of the ACM/IEEE international conference on Humanrobot interaction, 2007, 49-56

[17] Saunders, J.; Nehaniv, C. \& Dautenhahn, K. Teaching robots by moulding behavior and scaffolding the environment. Proceedings of the 1st ACM SIGCHI/SIGART conference on Human-robot interaction, 2006, 118-125

[18] Thomaz, A. L. \& Breazeal, C. Teachable robots: Understanding human teaching behavior to build more effective robot learners Artif. Intell., Elsevier Science Publishers Ltd., 2008, 172, 716-737

[19] Thomaz, A. L. \& Cakmak, M. Learning about objects with human teachers. HRI '09: Proceedings of the 4th ACM/IEEE international conference on Human robot interaction, ACM, 2009, 15-22

[20] Thomaz, A.; Hoffman, G. \& Breazeal, C. Experiments in socially guided machine learning: understanding how humans teach. Proceedings of the 1st ACM SIGCHI/SIGART conference on Humanrobot interaction, 2006, 359-360

[21] Veeraraghavan, H. \& Veloso, M. Teaching sequential tasks with repetition through demonstration. Proceedings of the 7th international joint conference on Autonomous agents and multiagent systemsVolume 3, 2008, 1357-1360

[22] Vygotsky, L. S. Mind in society: the development of higher psychological processes. Harvard University Press, Cambridge, MA, 1978.

[23] Watson, D.; Clark, L. \& Tellegen, A. Development and validation of brief measures of positive and negative affect: the PANAS scales. Journal of personality and social psychology, J Pers Soc Psychol, $1988,54,1063$

[24] Weiss, A., Bernhaupt, R., Lankes, M., and Tscheligi, M. The usus evaluation framework for human-robot interaction. In AISB2009: Proceedings of the Symposium on New Frontiers in Human-Robot Interaction, 2009, accepted for publication. 